\title{
A Novel Heart Rate Robust Method for Short-Term Electrocardiogram Biometric Identification
}

\author{
Di Wang ${ }^{1}$, Yujuan Si ${ }^{1,2}$, , Weiyi Yang ${ }^{1}$, Gong Zhang ${ }^{1}$ and Tong Liu ${ }^{3}$ \\ 1 College of Communication Engineering, Jilin University, Changchun 130012, China; \\ wangdi17@mails.jlu.edu.cn (D.W.); yangwy17@mails.jlu.edu.cn (W.Y.); zhanggong18@mails.jlu.edu.cn (G.Z.) \\ 2 School of Electronic and Information Engineering (SEIE), Zhuhai College, Jilin University, Zhuhai 519041, \\ China \\ 3 School of Information and Electrical Engineering, Ludong University, Yantai 264025, China; liut@ldu.edu.cn \\ * Correspondence: siyj@jlu.edu.cn
}

Received: 8 December 2018; Accepted: 3 January 2019; Published: 8 January 2019

check for updates

\begin{abstract}
In the past decades, the electrocardiogram (ECG) has been investigated as a promising biometric by exploiting the subtle discrepancy of ECG signals between subjects. However, the heart rate (HR) for one subject may vary because of physical activities or strong emotions, leading to the problem of ECG signal variation. This variation will significantly decrease the performance of the identification task. Particularly for short-term ECG signal without many heartbeats, the hardly measured HR makes the identification task even more challenging. This study aims to propose a novel method suitable for short-term ECG signal identification. In particular, an improved HR-free resampling strategy is proposed to minimize the influence of HR variability during heartbeat processing. For feature extraction, the Principal Component Analysis Network (PCANet) is implemented to determine the potential difference between subjects. The proposed method is evaluated using a public ECG-ID database that contains various HR data for some subjects. Experimental results show that the proposed method is robust to HR change and can achieve high subject identification accuracy (94.4\%) on ECG signals with only five heartbeats. Thus, the proposed method has the potential for application to systems that use short-term ECG signals for identification (e.g., wearable devices).
\end{abstract}

Keywords: ECG identification; short-term ECG signals; HR-free resampling strategy; principal component analysis network; ECG-ID

\section{Introduction}

Biometric systems play an important role in security applications and have been deployed around the world in past decades. Currently, common used biometrics in practice include face, fingerprint, iris, etc. However, neither can these biometrics effectively avoid being stolen, nor are they robust enough to falsification. For instance, with finger marks left behind on objects, crackers can recreate fingerprint using latex; Iris images can be captured in a few meters distance and falsified by using contact lenses with copied iris feature printed on [1]; Biometric systems utilizing facial recognition can be easily fooled by high-resolution still photos. In recent years, it has been observed that electrocardiogram (ECG) is a significant signature for individuals even within siblings or twins [2]. Unlike other biometrics, ECG is an inner signal, whose presence automatically ensures the liveness [3,4]. This property makes ECG far more difficult to be stolen or falsified.

The ECG signal is the recording of periodic variation with heart beating. A heartbeat represents one period of the ECG signal, which conveys rich identity information and is an important sign for subject identification. However, visual interpretation of beats is difficult because the changes 
in amplitude and duration are subtle. To deal with this problem, pattern recognition methods are preferred in ECG identification due to their reliable, quick, and objective analysis.

Within the last decade, many methods, based on neural networks [5,6], support vector machine [7], and k-nearest neighborhood [8] have been proposed in literature for the purpose of automatic identification. The literature [9] presented a recurrent neural network (RNN)-based method, which could achieve automatic feature extraction, to improve the identification performance on ECG signals from both the same session and different sessions. Discrete wavelet transform was used to extract wavelet coefficients as the feature vector and $\mathrm{KNN}$ was applied as the classifier in literature [10]. A novel automatic ECG identification approach combining back propagation neural network (BP-NN) with Frequency Rank Order Statistics (FROS) was introduced to distinguish different subjects in the literature [11]. The literature [12] utilized neural networks to both identify QRS complex segments and perform user authentication on these segments. All these methods mainly focus on the development of the part of feature extraction and classification. By properly combing the approaches of feature extraction with improved classifiers, these methods have achieved good performance. However, it is noted that most of them are evaluated on signals with stable heart rate (HR). Actually, there are the variation of ECG signals of one person due to HR change. For example, HR increase will shorten the duration of the ventricular depolarization period, leading to T wave shift. In other ECG applications, such as atrial fibrillation (AF) [13], the HR variability can be used to distinguish the AF episode from normal sinus rhythm. However, in ECG biometric, this variation will result in low identification task and make the identification become far more difficult without appropriate processing [14].

To overcome this limitation, a popular method is to normalize QT interval according to correction formulas. For example, Lugovaya et al. [15] scaled the ST-fragment based on Framingham and Bazett's formulas. Francesco et al. [16] preferred to use a different formula based on the suggestion presented by Tawfik in [17]. Besides methods based on QT correction, Kiran et al. [18] also proposed an effective feature extraction depending on the characteristic points, which were $\mathrm{P}, \mathrm{Q}, \mathrm{R}, \mathrm{S}$, and T. By taking less HR related parameters as features, this method was least affected by HR change. However, the performance of these above methods depends on the accurate localization of wave boundaries for QT interval estimation or HR measurement. Though the $\mathrm{R}$ and $\mathrm{T}$ wave detection techniques have started to provide acceptable results in most cases, detecting $P, Q$, and $S$ is still challenging [19]. Furthermore, HR measurement of ECG signals requires multiple ECG signals and may not be desirable for systems that use short-term ECG signals for identification. Thus non-fiducial or partial-fiducial identification methods should be investigated.

To solve this problem, Wonki Lee et al. [20] proposed a novel partial-fiducial method, whose idea was that one heartbeat was resampled and mapped into a regular interval by ignoring temporal period information. Taking a pattern matching algorithm based on Euclidean distance as the classifier, they achieved a maximum performance of $98.36 \%$ accuracy using finger ECG data. However, according to the research of Mikhail Matveev1 et al. [21], QRS negative area, total area, slope from R to S peak and sum of the absolute QRS velocities values have a strong correlation between ECG recordings acquired 5 years apart. These features will be distorted during the resampling process proposed by [20], hindering a further increase of identification accuracy. Identification performance can be potentially improved by appropriately reserving the original information of the QRS complex.

The aim of this study is to propose an HR robust ECG identification method suitable for short-term ECG signals. Our work contains two main parts: (1) On the basis of the research of reference [20], we propose a QRS-centered resampling strategy for heartbeat processing. The method aims to completely preserve the original temporal and morphological information of the QRS complex while solving the problem of $\mathrm{T}$ wave shift. As a result, more potentially helpful information with less HR influence will be provided for the subsequent steps; (2) For feature extraction, Convolution Neural Network $(\mathrm{CNN})$ has proven its effectiveness in medical research such as health informatics and computed tomography image analysis in recent years [22-25], e.g., Acharya et al. [22] conducted a CNN study for automatic arrhythmia detection and recorded accuracy, sensitivity and specificity of $92.50 \%, 98.09 \%$, 
and $93.13 \%$, respectively, for two seconds of ECG segments. In our work, Principal Component Analysis Network (PCANet) proposed by Tsung-Han Chan et al. [26], a new kind of CNN that employs PCA to learn multistage filter banks, is adopted as the tool to mine more useful components from the processed heartbeats. By using PCA filters as the convolution kernel, this network is sensitive to the local difference among heartbeats from different subjects. At last, a linear Support Vector Machine (linear-SVM) is used to address the identification for faster training and classification.

The rest of this paper is organized as follows: Section 2 illustrates the proposed methodology; Database and experimental results are shown in Section 3; the results of our approach are discussed in Section 4; and Section 5 concludes the paper.

\section{Methods}

The whole proposed identification process is mainly composed of five parts: (1) preprocessing; (2) HR variability removal; (3) feature extraction; (4) beat identification; and (5) subject identification. Figure 1 depicts the diagram of the ECG identification methodology proposed in this paper.

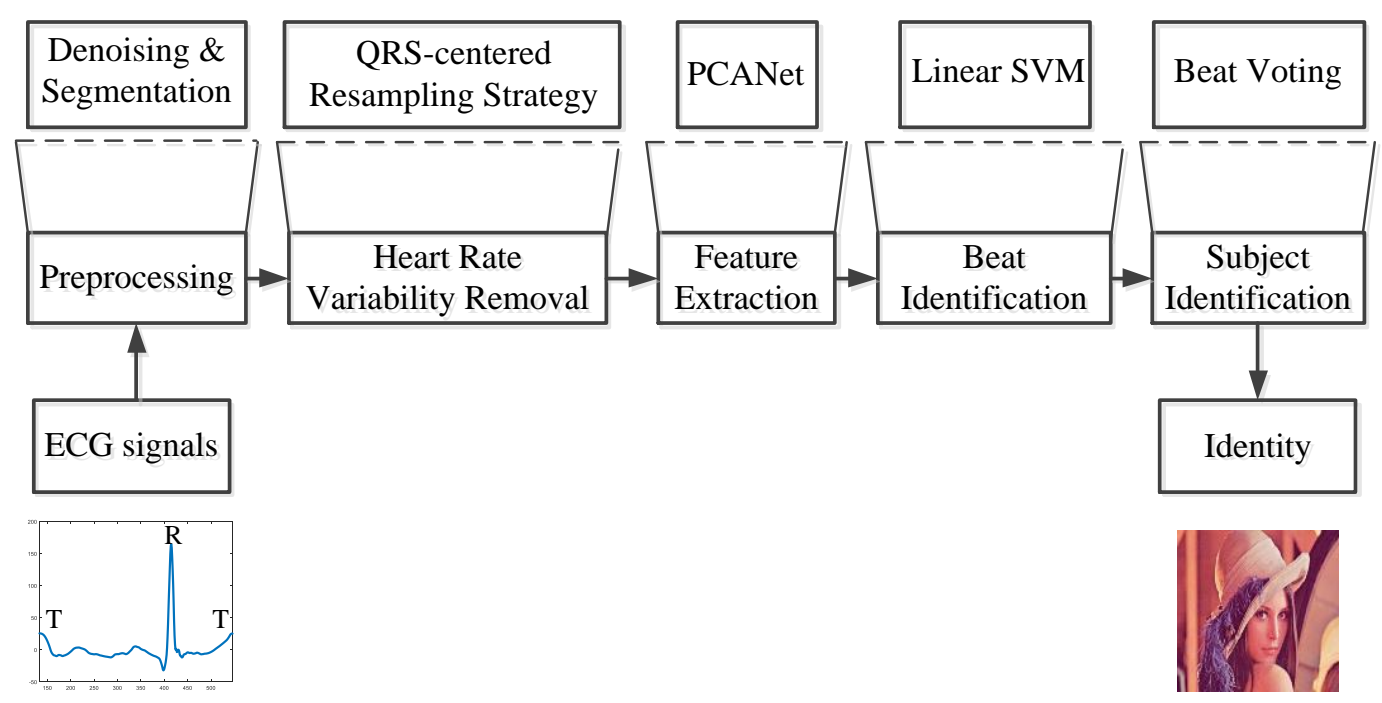

Figure 1. Diagram of electrocardiogram (ECG) identification methodology proposed.

\subsection{Preprocessing}

Denoising: Multiple factors will interfere with the quality of the ECG signal. The origins of interference are usually various. For example, during the acquisition of ECG signals, power-line interference generates because of the frequency influence of the used power. Interference will display in the form of noise and distort the waveform of ECG signals, leading to the decrease of ECG identification performance. Real raw ECG signals contain three major noise, namely, baseline drift, power-line interference and Electromyogram (EMG) artifact. Generally, the frequency of baseline drift is less than $0.5 \mathrm{~Hz}$ [27] and that of power-line interference is $50 \mathrm{~Hz}$ (or $60 \mathrm{~Hz})$ [28]. While EMG artifact is a random noise that spreads over the entire frequency range [29]. In this paper, wavelet transform (WT) is employed as the de-nosing method due to its property of sparsity, locality and multi-resolution [30]. The wavelet-based de-noising process is summarized as follows: Raw signals are decomposed to 9 levels by lifting wavelet transform with wavelet db4; Obtained detail coefficients of different levels are thresholded by shrinkage (soft) strategy; Reconstructing the original sequence from the thresholded wavelet detail coefficients leads to removal of noise. Here the shrinkage strategy uses the universal 'VisuShrink' threshold given by [31]:

$$
T h r=\sigma \sqrt{2 \log (N)}
$$


where $N$ is the number of data points and $\sigma$ represents the estimated noise level, which is obtained according to [32]:

$$
\sigma_{i}=\frac{\operatorname{median}\left(\left|\omega_{i}\right|\right)}{0.6745}
$$

where $\sigma_{i}$ is the noise level of the $i$-th level and median $(x)$ can output the median value of input sequence $x$. The functions lwt, wthresh, and ilwt in MATLAB were employed as the method for wavelet decomposition, coefficient thresholding and signal reconstruction respectively. Figure 2 shows the comparison between the original signal and the denoised signal.

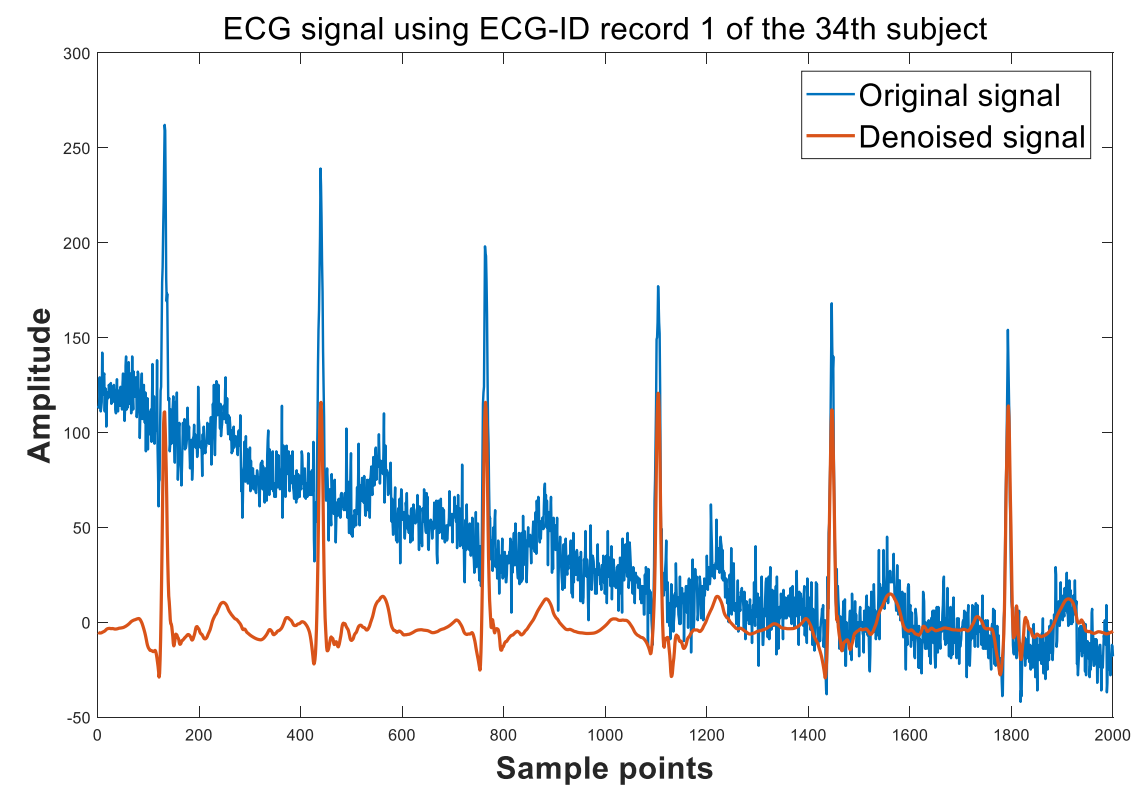

Figure 2. Comparison between the original signal and the de-noising signal.

Segmentation: After denoising, the $\mathrm{R}$ and $\mathrm{T}$ peak detection task is performed on denoised signals by using the ECGPUWAVE tool box [33]. Then the detected peak points at $\mathrm{T}$ are taken as the delimiters for segmentation. Figure 3 shows a beat extracted in the T-T way. It can be seen that the beat starts with its former $\mathrm{T}$ peak and ends with its own $\mathrm{T}$ peak. The duration is exactly a cardiac cycle.

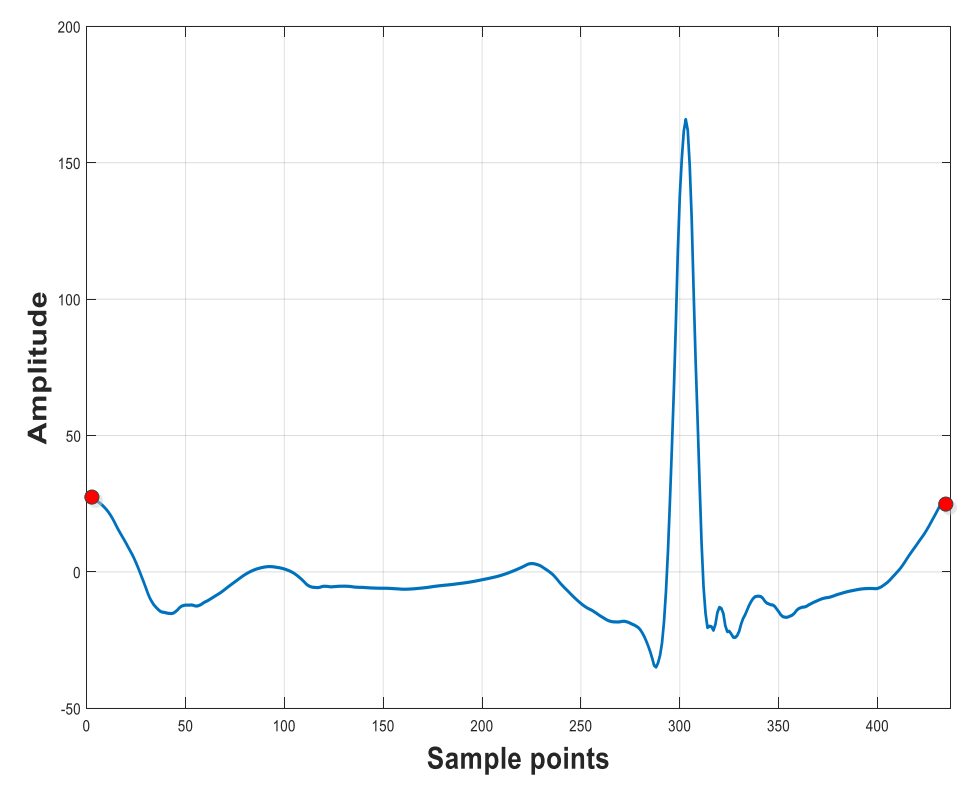

Figure 3. An extracted heart beat taking $\mathrm{T}$ as the delimiters. 


\subsection{QRS-Centered Resampling Strategy}

As mentioned above, an ECG signal can be segmented into several heartbeats. Comparison of original and processed heartbeats is shown in Figure 4. Figure 4a shows several beats extracted by taking T peaks as delimiter. It is noted that even beats $\alpha, \beta$, and $\gamma$, which comes from the same subject, do not have the same ECG waveform pattern. This nonstandard format cannot satisfy the requirement of subsequent pattern matching, thus framing an arbitrary length of beat into a regular interval of the same length is necessary.

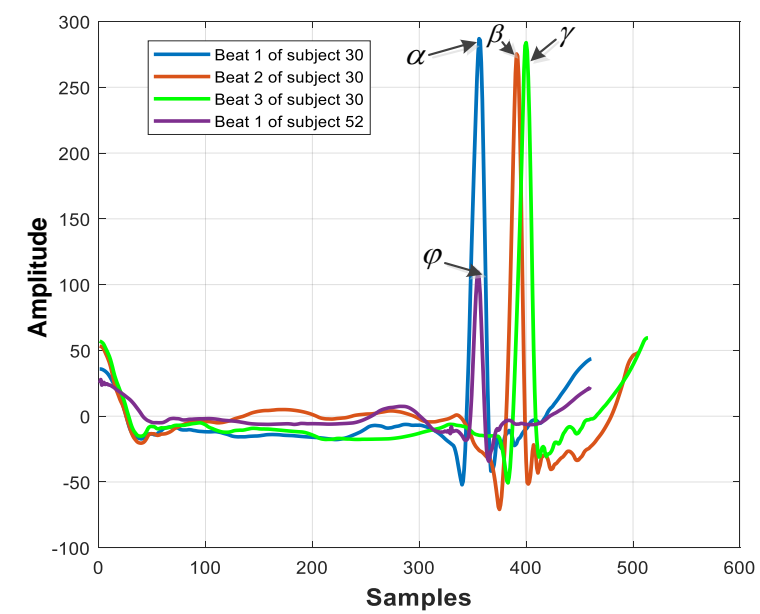

(a)

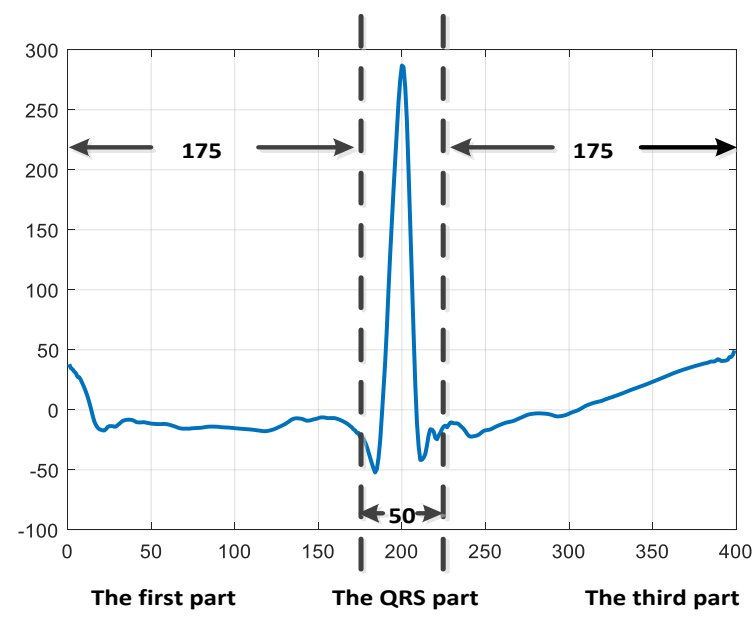

(c)

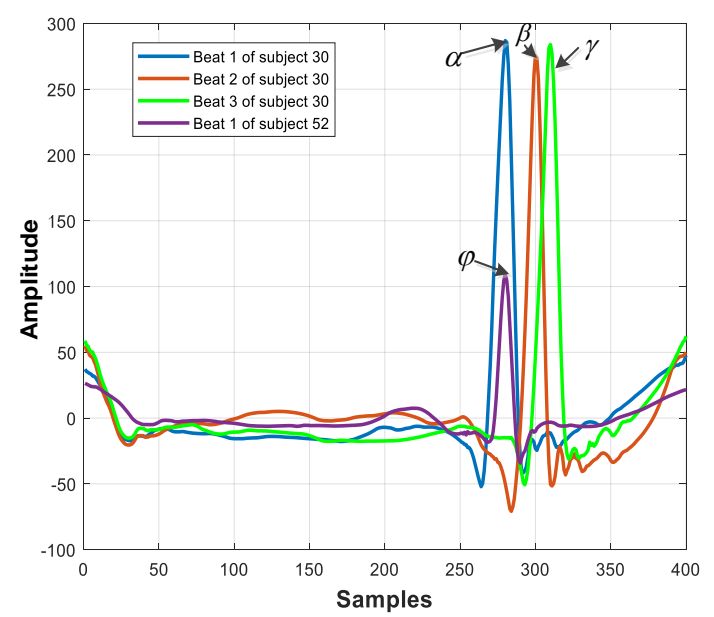

(b)

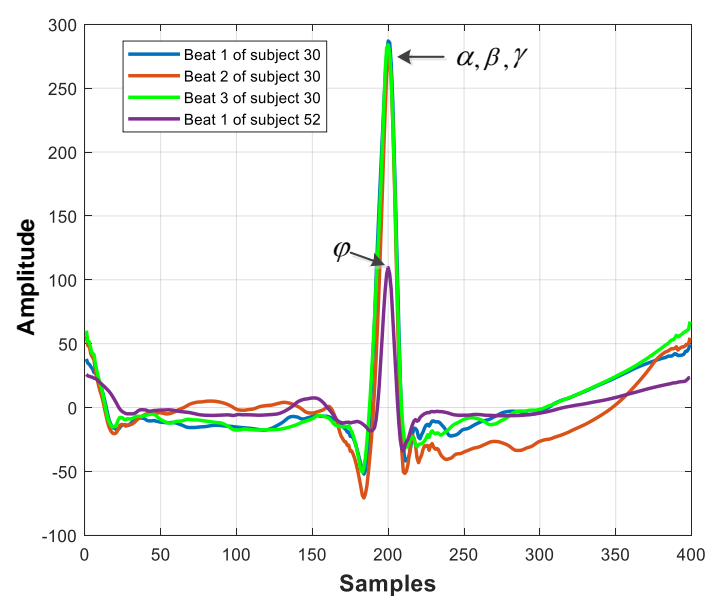

(d)

Figure 4. Comparison of heartbeats with different resampling manners. (a) Original heartbeats; (b) Heartbeats with direct resampling; (c) Normalization progress of the QRS-centered resampling; and (d) Heartbeats processed by the proposed method.

Generally, resampling-process [34] can provide a sufficient way for format standardization. Heartbeats with direct resampling can be seen in Figure $4 \mathrm{~b}$. It is noted that QRS waveforms of beats $\alpha$, $\beta$, and $\gamma$ are similar to each other, but locate in different positions on the beats. Beat $\varphi$ has a different QRS waveform from the above three, but its position is similar to $\alpha$. As a result, it is likely found that the similarity measurements among $\alpha, \beta$, and $\gamma$ are larger than that between $\alpha$ and $\varphi$, leading the following decision to an opposite result.

To solve this problem, a QRS-centered resampling strategy is proposed in this section. Based on the research of $[5,20,21]$, our idea is that any temporal and morphological information of the QRS complex may potentially contribute to ECG identification. Meanwhile, only the amplitude is useful 
in identifying a subject for the rest part. Thus, we remain QRS complex original to preserve all QRS information and resample the rest part to correct $\mathrm{T}$ wave shift by ignoring its temporal information. Figure $4 \mathrm{c}$ shows the proposed normalization progress. In our strategy, each heartbeat is considered as three parts, namely, the first part, the QRS part and the third part. The first part is from the start-point of beat to the start-point of the QRS part; the QRS part is centered on the detected R point and has unified 50 points; the third part is from the end-point of the QRS part to the end-point of beat. The process of the proposed strategy is summarized as follows: Firstly, considering that the QRS duration of a healthy subject is generally $60 \sim 100 \mathrm{~ms}$, a 50-point width window (the digitalized frequency of ECG-ID is 500 $\mathrm{Hz}$ and $100 \mathrm{~ms}$ corresponds to 50 points on this database) centered on $\mathrm{R}$ point is used to determine boundaries of three parts on heartbeats; Then extracted QRS parts are aligned centered on R peaks; Lastly, resampling process is utilized to normalize the first and third part both to 175 points. Extracted beats with our strategy can be seen in Figure $4 \mathrm{~d}$. The morphology of beat $\alpha, \beta$, and $\gamma$, becomes more similar to each other, and shows a significant difference from beat $\varphi$ at the same time. The function resample of MATLAB is employed as the method for resampling.

\subsection{PCANet}

Principal Component Analysis Network (PCANet) is a simple deep learning method to extract high-level features from the original input. Unlike traditional deep learning networks such as Convolution Neural Network (CNN) or Deep Belief Network (DBN), it does not need complicated iterative process for numerous parameters optimization. Only a few network parameters are necessary for determining a PCANet. Once these parameters are fixed, training the PCANet will be extremely simple and efficient. Figure 5 shows the structure of PCANet for ECG feature extraction. Code implementation of PCANet is available on [35].

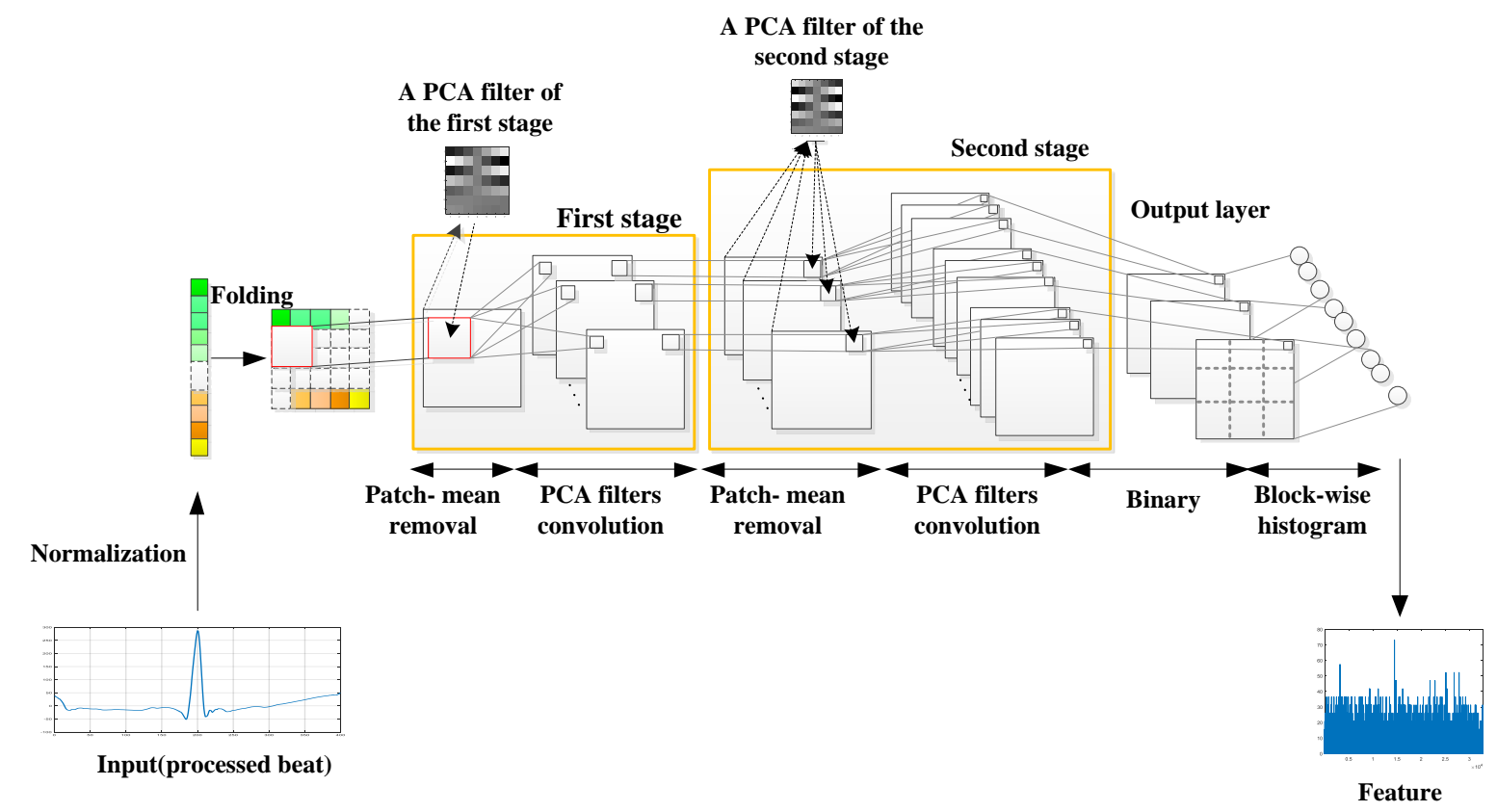

Figure 5. The structure of Principal Component Analysis Network (PCANet) model for ECG feature extraction.

\subsubsection{Normalization}

Given a heartbeat sample $x$ processed by QRS-centered resampling strategy, it should be normalized before feature extraction. Here, we use min-max normalization to realize this process. The function of mapminmax in MATLAB is employed as the method for normalization. 


\subsubsection{Input Layer}

The main function of the input layer is to fold the normalized heartbeat vectors into matrices, which are suitable for the use in the convolution process of PCANet. Assume the normalized heartbeat vector $x^{*}=\left[x_{1}^{*}, x_{2}^{*}, x_{3}^{*}, \cdots, x_{m n}^{*}\right]$, its specific folding process can be seen in Equation (3):

$$
\left[x_{1}^{*}, x_{2}^{*}, x_{3}^{*}, \cdots, x_{m n}^{*}\right] \rightarrow\left[\begin{array}{cccc}
x_{1}^{*}, & x_{2}^{*}, & \cdots, & x_{n}^{*} \\
x_{n+1}^{*}, & x_{n+2}^{*}, & \cdots, & x_{2 n}^{*} \\
\vdots & \vdots & \vdots & \vdots \\
x_{(m-2) n+1}^{*}, & x_{(m-2) n+2}^{*} & \cdots, & x_{(m-1) n}^{*} \\
x_{(m-1) n+1}^{*}, & x_{(m-1) n+2}^{*}, & \cdots, & x_{m n}^{*}
\end{array}\right]
$$

where $m n$ is the number of sampling point in the heartbeat vector. In this paper, since the dimension of nomalized heartbeat is 400, we set both $m$ and $n$ to 20. The function of reshape in MATLAB is employed as the method for folding.

\subsubsection{The First Stage (PCA)}

In this stage, we firstly use a $k_{1} \times k_{2}$ patch to scan one heartbeat matrix with step 1 to collect its all patches. Then we make each patch subtract the mean of all patches and obtain all mean-removal patch matrices of the heartbeat matrix. By constructing the same matrix for $N$ heartbeat matrices and combining them, we get their combination $Y$.

Then we perform Principal Component Analysis (PCA) on the combination Y. In this way, principal eigenvectors which are ordered based on the decrement of the corresponding eigenvalues can be obtained. By selecting the first $L_{1}$ principal eigenvectors and respectively reconstructing them to matrices with size $k_{1} \times k_{2}$, we can get the PCA filter bank of the first convolution layer. Equation (4) shows the specific process:

$$
W_{l}^{1}=\operatorname{mat}_{k_{1} k_{2}}\left(q_{l}\left(Y Y^{T}\right)\right) \in R^{k_{1} \times k_{2}}, l=1,2, \cdots, L_{1}
$$

where $W_{l}^{1}$ is the $l$-th PCA filter of the first convolution layer, $Y Y^{T}$ is the covariance matrix of $Y, q_{l}()$ extracts the $l$-th principal eigenvector of $Y Y^{T}$, and $m a t_{k_{1}, k_{2}}()$ maps the extracted principal eigenvector to a matrix $W \in R^{k_{1} \times k_{2}}$.

\subsubsection{The Second Stage (PCA)}

After obtaining PCA filters of the first layer, we can get the filter output by doing convolution on the heartbeat matrix and PCA filter $W_{l}^{1}$. Then the solution process of PCA filter banks in the second stage is just the same as that in the first stage. We firstly scan the first layer output using a patch with size $k_{1} \times k_{2}$ and collect a series of patches. Then the patch mean is subtracted from each patch and the mean-removed patches of the $l$-th filter output of all heartbeat matrix are combined together to obtain patch matrix. For all of the filter, their patch matrices are concatenated to get combination $\widetilde{Y}$. The PCA filters of the second stage are then obtained as shown in Equation (5):

$$
W_{\ell}^{2}=\operatorname{mat}_{k_{1}, k_{2}}\left(q_{\ell}\left(\tilde{Y} \widetilde{Y}^{T}\right)\right) \in R^{k_{1} \times k_{2}}, l=1,2, \cdots, L_{2}
$$

where $W_{\ell}^{2}$ is the $\ell$-th PCA filter of the second convolution layer, $\widetilde{Y} \widetilde{Y}^{T}$ is the covariance matrix of $\widetilde{Y}, q_{\ell}()$ extracts the $\ell$-th principal eigenvector of $\tilde{Y} \widetilde{Y}^{T}$, and mat $_{k_{1}, k_{2}}()$ maps the extracted principal eigenvector to a matrix $W \in R^{k_{1} \times k_{2}}$. For each input of the second stage, one will output $L_{2}$ matrices of size $m \times n$. The specific computation process is doing convolution on the input and its corresponding filter in the second stage. 


\subsubsection{Output Stage}

After the second stage, obtained outputs are firstly binarized by a Heaviside function. By this function, value in outputs will be 1 for positive entries and 0 otherwise. Then we convert the $L_{2}$ outputs back into a single integer-valued matrix by Equation (6):

$$
\Gamma_{i}^{l}=\sum_{\ell=1}^{L_{2}} 2^{\ell-1} H\left(y_{i}^{l} * W_{\ell}^{2}\right)
$$

where $y_{i}^{l}$ means the output of $l$-th filter for the $i$-th heartbeat matrix $y_{i}$ in the first layer. Here weights of the outputs are irrelevant since each integer is treated as a distinct "word". After coding, each integer-valued matrix $\Gamma_{i}^{l}$ is partitioned into B blocks with a set overlapping ratio $v$ and histogram block size $\eta$. We compute the histogram of the values in each block and concatenate all $B$ histograms into one vector which is denoted as Bhist $\left(\Gamma_{i}^{l}\right)$. The feature of input heartbeat vector is lastly defined to be the set of block-wise histograms as shown in Equation (7):

$$
f_{i}=\left[\operatorname{Bhist}\left(\Gamma_{i}^{1}\right), \cdots, \operatorname{Bhist}\left(\Gamma_{i}^{L_{1}}\right)\right]^{T} \in R^{\left(2^{L_{2}}\right) L_{1} B}
$$

where $f_{i}$ is the learned feature representation which can be used in following classification.

The detailed information of network parameter setting can be seen in Table 1.

Table 1. Detailed parameter information of PCANet used for ECG identification.

\begin{tabular}{ccc}
\hline Steps & Project & Parameter \\
\hline Input & Heartbeat matrix size & $20 \times 20$ \\
The first stage & Patch size $\left(k_{1} \times k_{2}\right)$ & $7 \times 7$ \\
& The number of filters of the first stage $(l)$ & 8 \\
The second stage & Patch size $\left(k_{1} \times k_{2}\right)$ & $7 \times 7$ \\
& The number of filters of the second stage $(\ell)$ & 8 \\
Output & Histogram block size $(\eta)$ & $7 \times 7$ \\
& Block overlap ratio $(v)$ & 0.5 \\
\hline
\end{tabular}

\subsection{Classifier}

Several classifiers such as K-Nearest Neighbors (KNN), Back propagation neural network (BP-NN), Random Forest (RF), Naive Bayes and Support Vector Machines (SVM) are implemented and compared. The detail of these classifiers is presented below.

\subsubsection{K-Nearest Neighbor (KNN)}

In pattern recognition, k-nearest neighbor algorithm is a common method used for both classification and regression. It is a type of instance-based learning, or lazy learning. It does not attempt to construct a general model, but simply stores instances of the training data. Classification is computed from a simple majority vote of the nearest neighbors of the instance to be predicted. A query instance is assigned the data class which has the most representatives within the nearest neighbor of the instance. Here, we used Euclidean distance as our distance metric. In our experiment, the best result of classification was achieved for $\mathrm{K}=3$. $\mathrm{KNN}$ can be realized by the function ClassificationKNN.fit in MATLAB.

\subsubsection{Back Propagation Neural Network (BP-NN)}

BP-NN is a multilayer feed forward network trained with error back propagation strategy. It is firstly proposed by Rumelhart [36] and has been extensively used at present. In this paper, we used a typical three-layer BP-NN as classifier to classify the extracted features, and the number of units in 
the hidden layer was set to 50. The function patternnet in MATLAB was employed as the method for BP-NN implementation.

\subsubsection{Random Forest (RF)}

A random forest model [37] is a collection of $k$ decision trees. Here, cart classification trees, which divide attributes based on the Gini index, are developed with different numbers of inputs to form an RF. The classification is determined by the voting results of all decision trees, and the highest ranked class is selected as the final label of a new instance. In our experiment, the best classification results occurred when $k$ was set to 500. RF model was implemented by using the function TreeBagger in MATLAB.

\subsubsection{Naive Bayes Classifier}

Naive Bayes classifier is one of the simplest machine learning algorithms, being also fast and easy to implement. It is a probabilistic classifier based on applying Bayes' theorem with strong independence assumptions between the features, and has proven to work surprisingly well in practice. In this work, a Naive Bayes classifier using Bernoulli distribution was adopted for heartbeat identification. Code implementation of Naive Bayes classifier is available on [38].

\subsubsection{Support Vector Machines (SVM)}

Support Vector Machine (SVM) is one of the state-of-the-art classifiers which can split a dataset into two or more categories. By using a function called kernel, support vector machine can transform the input samples into a higher dimensional space and classify them linearly. In this paper, since features extracted by PCANet are high-dimensional and sparse, we adopt a linear kernel support vector machine (linear-SVM) according to [25]. Linear-SVM can be realized by freely available Liblinear toolkit [39]. During the use of Liblinear, parameter $C$ known as error penalty factor, which expresses the tolerance to error, was set to 1 for good performance of classifier.

\subsection{Signal Identification}

With the features extracted by PCANet, linear-SVM can output the identification result of each beat. To get the label of a whole signal, we make beats of the same signal vote. According to the results, the class with maximum number of votes is selected as the class label of the estimated signal.

\section{Results}

\subsection{Database}

We use the challenging ECG-ID database [15], which is available on the PhysioNet, to evaluate our proposed method. The database is chosen because it includes more than two recordings for some of its subjects. All recordings in this database are acquired in lead I and digitalized at $500 \mathrm{~Hz}$ over a duration of $20 \mathrm{~s}$. Unlike such databases as Massachusetts Institute of Technology-Biotechnology arrhythmia database (MIT-BIH-AHA), whose signals have stable HR, the HR of signals in the ECG-ID is various. Different emotional or physical conditions and acquisition over a large domain of time make it provide a platform for use of ECG as person identification in real world scenarios. In the experiments, we select the same 12 subjects as reference [18] and each subject has five recordings for training and testing purposes. Table 2 shows the detail information of each recording, including its contained heartbeat number and estimated HR. HR is estimated as follows:

$$
H R=(\text { Heartbeat Number }) /(\text { Signal Duration }) * 60
$$

here since the signal duration is $20 \mathrm{~s}, \mathrm{HR}$ is three times as much as contained Heartbeat Number. 
Table 2. Heart number \& estimated heart rate (HR) of used signals for experiments.

\begin{tabular}{|c|c|c|c|c|c|c|c|c|c|c|}
\hline \multirow{3}{*}{$\begin{array}{c}\text { Subject Number } \\
3\end{array}$} & \multicolumn{10}{|c|}{ Heartbeat Number \& Estimated Heart Rate (beat/min) } \\
\hline & \multicolumn{2}{|c|}{ Record-1 } & \multicolumn{2}{|c|}{ Record-2 } & \multicolumn{2}{|c|}{ Record-3 } & \multicolumn{2}{|c|}{ Record-4 } & \multicolumn{2}{|c|}{ Record-5 } \\
\hline & 25 & 75 & 25 & 75 & 24 & 72 & 23 & 69 & 23 & 69 \\
\hline 10 & 28 & 84 & 27 & 81 & 28 & 84 & 33 & 99 & 24 & 72 \\
\hline 24 & 24 & 72 & 25 & 75 & 25 & 75 & 21 & 63 & 18 & 54 \\
\hline 25 & 24 & 72 & 24 & 72 & 23 & 69 & 19 & 57 & 23 & 69 \\
\hline 30 & 23 & 69 & 24 & 72 & 21 & 63 & 21 & 63 & 19 & 57 \\
\hline 32 & 22 & 66 & 22 & 66 & 23 & 69 & 24 & 72 & 23 & 69 \\
\hline 34 & 31 & 93 & 30 & 90 & 27 & 81 & 29 & 87 & 30 & 90 \\
\hline 36 & 19 & 57 & 20 & 60 & 23 & 69 & 25 & 75 & 23 & 69 \\
\hline 52 & 25 & 75 & 26 & 78 & 28 & 84 & 29 & 87 & 31 & 93 \\
\hline 53 & 26 & 78 & 27 & 81 & 27 & 81 & 30 & 90 & 27 & 81 \\
\hline 59 & 25 & 75 & 28 & 84 & 33 & 99 & 21 & 63 & 20 & 60 \\
\hline 72 & 24 & 72 & 25 & 75 & 20 & 60 & 37 & 111 & 34 & 102 \\
\hline
\end{tabular}

\subsection{Experimental Setup}

\section{Experiment 1}

As KNN, BP-NN, RF, Naive Bayes, and Linear-SVM have been widely used, these methods were implemented for evaluating the performance of the extracted features. To convincingly estimate the proposed method, two of the five ECG signals from the subject were combinatorially selected as the training set, and the remaining three were utilized as the testing set. As a result, each experiment was repeated ten $\left(C_{5}^{2}\right)$ times.

\section{Experiment 2}

In Experiment 2, the HR robustness was evaluated by comparing the proposed method with other five methods. During identification, all the six methods had the same denoising and fiducial point detection process, and the difference among them was the manner of heartbeat resampling and feature extraction. Six methods are shown in Table 3, where " $Y$ " indicates adoption and "N" indicates none. In heartbeat resampling manner, "QRS-centered" represents the proposed novel resampling strategy, and the meaning of "TT" and "TRT" is explained below.

Table 3. Six Methods with different resampling strategy and feature extraction manner.

\begin{tabular}{cccccccc}
\hline \multicolumn{2}{c}{ Main Operating } & TT-CNN & TRT-CNN & QRS-CNN & TT-PCANet & TRT-PCANet & $\begin{array}{c}\text { Proposed } \\
\text { Method }\end{array}$ \\
\hline \multirow{2}{*}{ Heartbeat } & TT & Y & N & N & Y & N & N \\
Resampling Manner & TRT & N & Y & N & N & Y & N \\
\multirow{2}{*}{ Feature Extraction } & QRS-centered & N & N & Y & N & N & Y \\
& PCANet & Y & Y & Y & N & N & N \\
\hline
\end{tabular}

"TT"-resampling: Segmented heartbeats with different sizes are directly resampled to 400 sample points without alignment of $\mathrm{R}$ points. This manner just performs a forced alignment of $\mathrm{T}$ wave points, ignoring all temporal period and morphological information of heartbeats.

"TRT"-resampling: $\mathrm{R}$ and $\mathrm{T}$ points of segmented heartbeats are aligned, and divide each heartbeat into two parts: T-R and R-T. Then both T-R and R-T part are resampled to 200 to obtain the unified size as in Method 1. Compared with Method 1, this method further performs alignment of key fiducial points (R). It is firstly proposed by reference [20] and has proved its effectiveness on heart robustness without requiring HR measurement. All the experiments are repeated 10 times.

Here the architecture of the used traditional CNN is shown in Table 4. 
Table 4. Detailed parameter information of traditional Convolution Neural Network (CNN) used for ECG identification.

\begin{tabular}{ccccc}
\hline Layers & Type & Number of Neurons (Output Layer) & Kernel Size & Stride \\
\hline $0-1$ & Convolution & $16 \times 16 \times 6$ & $5 \times 5$ & 1 \\
$1-2$ & Max-pooling & $8 \times 8 \times 6$ & $2 \times 2$ & 2 \\
$2-3$ & Convolution & $4 \times 4 \times 12$ & $5 \times 5$ & 1 \\
$3-4$ & Max-pooling & $2 \times 2 \times 12$ & $2 \times 2$ & 2 \\
\hline
\end{tabular}

\section{Experiment 3}

To evaluate the effectiveness of the proposed method on short-term ECG signals, original signals in test set were segmented into hundreds of fragments with three schemes, in which a single fragment contained one, three and five heartbeats respectively. Based on these obtained short-term ECG segmentation, the subject identification accuracies were achieved and compared.

4. Experiment 4

To further validate the proposed method, the experimental results of reference [18] were compared with ours in Experiment 3. Many performance parameters such as sensitivity, specificity, precision, and F1-score were calculated and compared based on the obtained confusion matrix. For fair comparison we followed the same data distribution as reference [18], in which only Record- 1 and Record-2 of each subject were employed as the training set.

\section{Experiment 5}

Further validation was performed by comparing the results of different methods in reference [9], namely, RNN, Gated Recurrent Unit (GRU), and Long Short-Term Memory (LSTM), with that of our method. Following reference [9], we evaluated the proposed method on two public databases, which were ECG-ID and MIT-BIH Arrhythmia database (MITDB). Here, the used subject number of ECG-ID database increased from 12 to 89 . The MITDB is a patient information database, which contains 47 subjects and can be employed to evaluate the proposed method on the level of patient. For each subject of the MITDB, five fragments of 18-heartbeat length, which were recorded at different time, were randomly extracted for training and testing purpose. Thus a five-fold cross validation could be performed for evaluation.

All the above experiments were made in MATLAB 2017a (MATLAB, 2017a, MathWorks, Natick, MA, USA).

\subsection{Experimental Results}

\subsubsection{Experiment 1}

Figure 6 gives the comparison of different classifiers fed by features extracted using the proposed method. Related to Figure 6, the features extracted by the QRS-centered resampling strategy and PCANet could produce high heartbeat and subject identification accuracy of $83.14 \%$ and $94.72 \%$ even with the simplest classifier KNN. Meanwhile, all the other classifiers could yield heartbeat accuracy over $85 \%$ and subject identification accuracy over $95 \%$. The obtained results demonstrated that the extracted features could reflect the difference between different subjects, and were effective for ECG identification. In our subsequent experiments, we selected Linear-SVM as the classifier not only because its performance was better compared with other classifiers, but also because it is more suitable for dealing with the extracted high-dimensional features. 


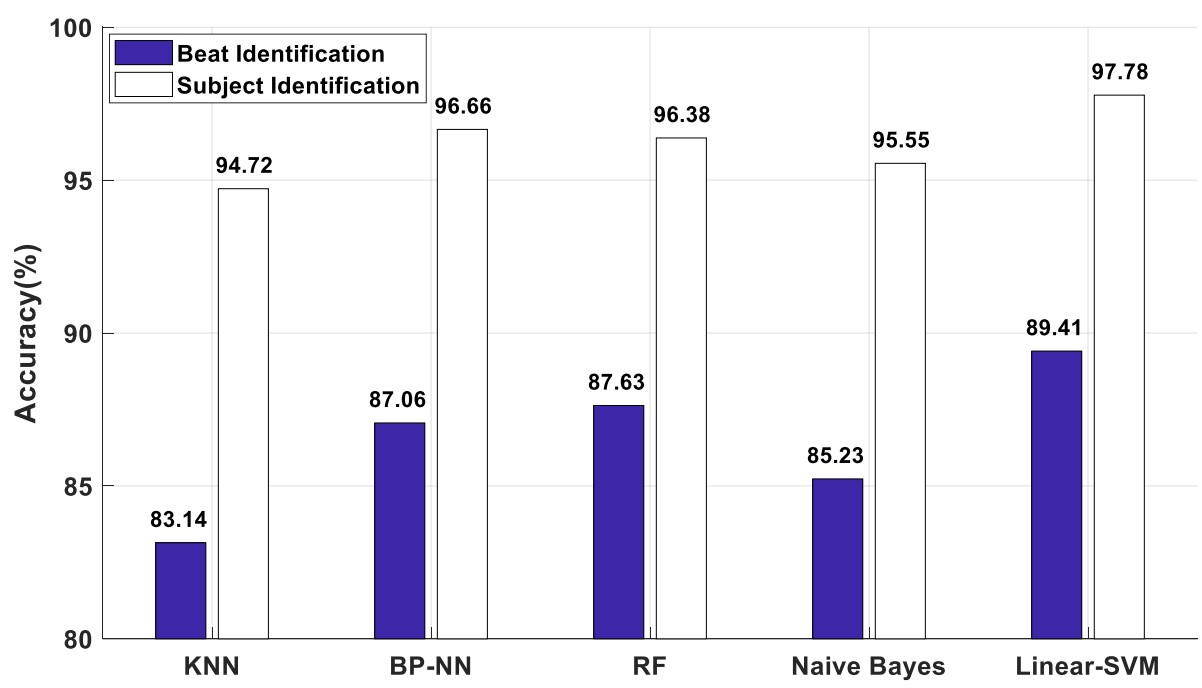

Figure 6. Comparison of different classifiers using the proposed method.

\subsubsection{Experiment 2}

Figure 7 shows the results of average heartbeat and subject identification accuracy of 10 experiments with six different methods, which are TT-CNN, TRT-CNN, QRS-CNN, TT-PCANet, TRT-PCANet, and the proposed method. Here the subject identification accuracy was obtained after all heartbeats of each signal voted. With TT-CNN and TT-PCANet, the average heartbeat (subject) identification rates were $71.51 \%(89.44 \%)$ and $77.22 \%(89.72 \%)$, respectively. They had the worst performance among the tested methods since it preserved the least information during heartbeat processing. The average accuracies increased to $77.86 \%$ (93.88\%) and 85.93\% (95.27\%) with TRT-CNN and TRT-PCANet, in which the R point alignment was additionally taken into account. The proposed method and QRS-CNN showed performance of $83.44 \%(94.4 \%)$ and $89.41 \%$ (97.78\%), respectively. It was found that when the feature extraction manner was set, methods with the proposed QRS-centered resampling strategy obtained the best heartbeat and subject identification, indicating that the preservation of the temporal period and morphological information of the QRS did benefit for identification accuracy. Also when compared with the CNNs, the PCANets achieved better performance using different heartbeat resampling manner. Furthermore, the CNNs $(6 \mathrm{c}-2 \mathrm{~s}-12 \mathrm{c}-2 \mathrm{~s})$ used in our work needed complicated iterative process for $468(5 \times 5 \times 6+6+5 \times 5 \times 12+12=468)$ parameters optimization, while the number of PCANets parameters is six $\left(k_{1}, k_{2}, l, \ell, \eta, v\right)$ here. Based on the results, we confirmed that our proposed method could achieve good performance under HR variability conditions.

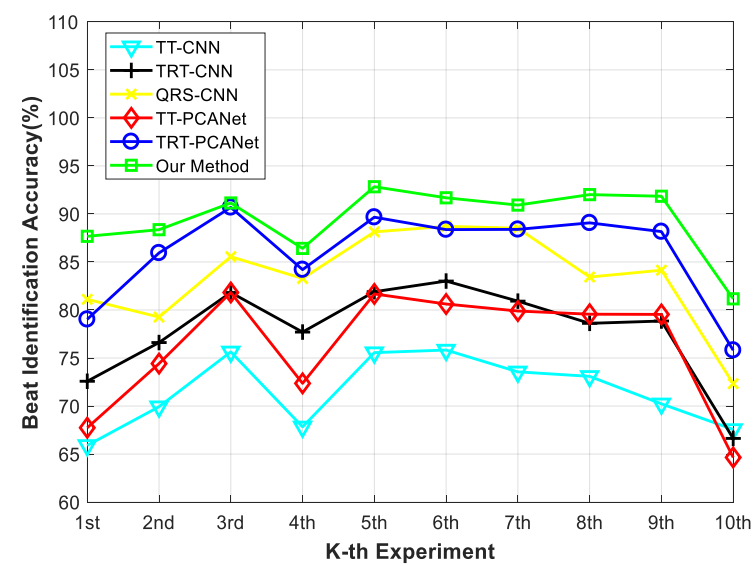

(a)

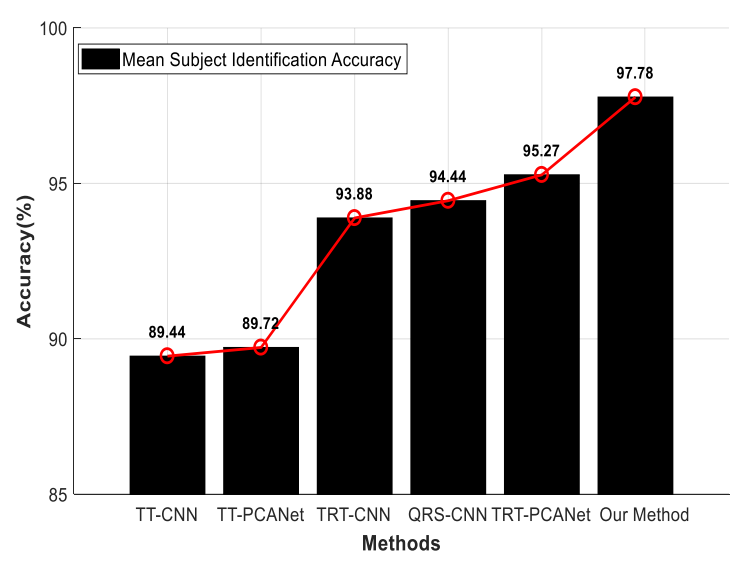

(b)

Figure 7. The accuracy comparison of the six identification methods. (a) Heartbeat identification comparison; (b) Comparison of mean subject identification accuracy of six methods. 


\subsubsection{Experiment 3}

Table 5 shows the variation of average subject identification accuracy with respect to different-length signals during ECG identification using six methods. Compared with the other five methods, higher identification accuracies were achieved by the proposed method under different conditions. The results also showed that the proposed method was able to get high accuracy over $94 \%$ even with signals of only five-heartbeat length. Nevertheless, for the rest methods, ECG signals should contain far more heartbeats to make the methods obtain the same accuracy. Generally, the goodness of a heartbeat based method in short-term ECG signal identification is mainly measured by the single heartbeat identification accuracy. Our method gave higher heartbeat identification accuracy of $89.41 \%$ on signals than the other five methods, which suggested that our method could provide an efficient way for short-term ECG identification $[40,41]$.

Table 5. The comparison of subject accuracies among short-term ECG signals with different heartbeat length.

\begin{tabular}{ccc}
\hline Method & $\begin{array}{c}\text { ECG Length } \\
\text { (in Number of Heartbeats) }\end{array}$ & $\begin{array}{c}\text { Average Subject Identification } \\
\text { Accuracy }\end{array}$ \\
\hline TT-CNN & 1 & $71.51 \%$ \\
TT-CNN & 3 & $77.57 \%$ \\
TT-CNN & 5 & $85.22 \%$ \\
TT-PCANet & 1 & $77.22 \%$ \\
TT-PCANet & 3 & $80.85 \%$ \\
TT-PCANet & 5 & $85.82 \%$ \\
TRT-CNN & 1 & $77.86 \%$ \\
TRT-CNN & 3 & $83.46 \%$ \\
TRT-CNN & 5 & $87.71 \%$ \\
TRT-PCANet & 1 & $85.93 \%$ \\
TRT-PCANet & 3 & $90.28 \%$ \\
TRT-PCANet & 5 & $91.96 \%$ \\
QRS-CNN & 1 & $83.44 \%$ \\
QRS-CNN & 3 & $88.96 \%$ \\
QRS-CNN & 5 & $91.89 \%$ \\
Our Method & 1 & $89.41 \%$ \\
Our Method & 3 & $92.49 \%$ \\
Our Method & 5 & $94.40 \%$ \\
\hline
\end{tabular}

\subsubsection{Experiment 4}

In this section, we compared the experimental results of [18] with ours to further validate the proposed method. Many performance parameters mentioned in [18] were calculated and compared based on the confusion matrix. The evaluation parameters include Accuracy, Sensitivity, Specificity, Precision and F1-score. The calculation process is as follows:

$$
\begin{gathered}
\text { Accuracy }=\frac{T P+T N}{T P+F P+T N+F N} \\
\text { Sensitivity }=\text { Recall }=\frac{T P}{T P+F N} \\
\text { Specificity }=\frac{T N}{T N+F P} \\
\text { Precision }=\frac{T P}{T P+F P} \\
F_{1} \text { Score }=\frac{2 * \text { Precision } * \text { Recall }}{\text { Precison }+ \text { Recall }}
\end{gathered}
$$

where TP, TN, FP, and FN, respectively represent True Positive, True Negative, False Positive, and False Negative. Since subject identification is a multi-class problem, this paper uses the overall statistic 
of the above indicators to evaluate the performance. The general calculation form of different overall statistics is as follows:

$$
\text { Overall_X }=\sum_{i=1}^{N} X_{i} / N_{\text {person }}
$$

where $N_{\text {person }}$ is the total number of evaluated person and $X_{i}$ represents the statistic result of the $i$-th person $\left(X_{i}\right.$ can be Accuracy, Recall, Specificity, Precision, or F1-score).

Table 6 shows the confusion matrix for the true classification of ECG beats used for testing the proposed method. The confusion matrix shows the number of beats used for the test with their corresponding True Positive Rate (TPR).

Table 6. Confusion matrix of true classification of ECG beat data of person.

\begin{tabular}{|c|c|c|c|c|c|c|c|c|c|c|c|c|c|}
\hline \multirow{2}{*}{ Target Class } & \multicolumn{12}{|c|}{ Classification Class } & \multirow{2}{*}{ Accuracy } \\
\hline & 3 & 10 & 24 & 25 & 30 & 32 & 34 & 36 & 52 & 53 & 59 & 72 & \\
\hline 3 & 19 & 0 & 1 & 0 & 0 & 1 & 0 & 0 & 0 & 0 & 0 & 0 & $90.48 \%$ \\
\hline 10 & 0 & 19 & 0 & 0 & 0 & 0 & 0 & 0 & 0 & 0 & 2 & 0 & $90.48 \%$ \\
\hline 24 & 3 & 1 & 16 & 1 & 0 & 0 & 0 & 0 & 0 & 0 & 0 & 0 & $76.19 \%$ \\
\hline 25 & 0 & 0 & 0 & 21 & 0 & 0 & 0 & 0 & 0 & 0 & 0 & 0 & $100 \%$ \\
\hline 30 & 0 & 0 & 0 & 0 & 21 & 0 & 0 & 0 & 0 & 0 & 0 & 0 & $100 \%$ \\
\hline 32 & 0 & 0 & 0 & 0 & 0 & 21 & 0 & 0 & 0 & 0 & 0 & 0 & $100 \%$ \\
\hline 34 & 2 & 0 & 0 & 1 & 0 & 0 & 18 & 0 & 0 & 0 & 0 & 0 & $85.71 \%$ \\
\hline 36 & 0 & 2 & 0 & 0 & 0 & 0 & 0 & 17 & 0 & 0 & 2 & 0 & $80.95 \%$ \\
\hline 52 & 0 & 0 & 0 & 0 & 0 & 0 & 0 & 0 & 21 & 0 & 0 & 0 & $100 \%$ \\
\hline 53 & 0 & 0 & 0 & 0 & 0 & 0 & 0 & 0 & 0 & 21 & 0 & 0 & $100 \%$ \\
\hline 59 & 0 & 0 & 0 & 0 & 0 & 0 & 0 & 0 & 1 & 0 & 17 & 3 & $80.95 \%$ \\
\hline 72 & 0 & 1 & 0 & 0 & 0 & 0 & 0 & 0 & 0 & 0 & 7 & 13 & $61.90 \%$ \\
\hline
\end{tabular}

With all heartbeats voting, we can obtain the result of subject identification. Table 7 shows the confusion matrix which includes the number of signals used for testing with their corresponding subject TPR. Based on the confusion matrices of heartbeat and subject identification, the evaluation parameters can be calculated and the results of comparison can be seen in Table 8 .

Table 7. Confusion matrix for true identification of person.

\begin{tabular}{cccccccccccccc}
\hline \multirow{2}{*}{ Target Class } & \multicolumn{10}{c}{ Classification Class } & \multirow{10}{*}{ Accuracy } \\
\cline { 2 - 9 } & $\mathbf{3}$ & $\mathbf{1 0}$ & $\mathbf{2 4}$ & $\mathbf{2 5}$ & $\mathbf{3 0}$ & $\mathbf{3 2}$ & $\mathbf{3 4}$ & $\mathbf{3 6}$ & $\mathbf{5 2}$ & $\mathbf{5 3}$ & $\mathbf{5 9}$ & $\mathbf{7 2}$ & \\
\hline 3 & $\mathbf{3}$ & 0 & 0 & 0 & 0 & 0 & 0 & 0 & 0 & 0 & 0 & 0 & $100 \%$ \\
10 & 0 & $\mathbf{3}$ & 0 & 0 & 0 & 0 & 0 & 0 & 0 & 0 & 0 & 0 & $100 \%$ \\
24 & 0 & 0 & $\mathbf{3}$ & 0 & 0 & 0 & 0 & 0 & 0 & 0 & 0 & 0 & $100 \%$ \\
25 & 0 & 0 & 0 & $\mathbf{3}$ & 0 & 0 & 0 & 0 & 0 & 0 & 0 & 0 & $100 \%$ \\
30 & 0 & 0 & 0 & 0 & $\mathbf{3}$ & 0 & 0 & 0 & 0 & 0 & 0 & 0 & $100 \%$ \\
32 & 0 & 0 & 0 & 0 & 0 & $\mathbf{3}$ & 0 & 0 & 0 & 0 & 0 & 0 & $100 \%$ \\
34 & 0 & 0 & 0 & 0 & 0 & 0 & $\mathbf{3}$ & 0 & 0 & 0 & 0 & 0 & $100 \%$ \\
36 & 0 & 0 & 0 & 0 & 0 & 0 & 0 & $\mathbf{3}$ & 0 & 0 & 0 & 0 & $100 \%$ \\
52 & 0 & 0 & 0 & 0 & 0 & 0 & 0 & 0 & $\mathbf{3}$ & 0 & 0 & 0 & $100 \%$ \\
53 & 0 & 0 & 0 & 0 & 0 & 0 & 0 & 0 & 0 & $\mathbf{3}$ & 0 & 0 & $100 \%$ \\
59 & 0 & 0 & 0 & 0 & 0 & 0 & 0 & 0 & 0 & 0 & $\mathbf{3}$ & 0 & $100 \%$ \\
72 & 0 & 0 & 0 & 0 & 0 & 0 & 0 & 0 & 0 & 0 & 2 & $\mathbf{1}$ & $33.33 \%$ \\
\hline
\end{tabular}

In the comparison of our method with seven beats and [18], our method showed improvement on all evaluation indicators. Results showed that the overall heartbeat and subject identification accuracy could reach $88.8889 \%$ and $99.0741 \%$, respectively. In terms of specificity, we achieved a prediction success average rate reaching $99.4949 \%$ and exceeding $95 \%$ for each person. Relative to the results of the previous method, our accuracy, precision, and F1-score were all at a high level. 
Table 8. Comparison of our method with seven beats and literature [18].

\begin{tabular}{cccc}
\hline S. No & Performance Parameter & Value [18] & Value (Our Method) \\
\hline 1 & True_Positive Rate (Beat Identification) & $80.5556 \%$ & $88.8889 \%$ \\
2 & True_Positive Rate (Subject Identification) & $88.8889 \%$ & $94.4444 \%$ \\
3 & Overall_Accuracy & $98.1481 \%$ & $99.0741 \%$ \\
4 & Overall_Recall & $88.8889 \%$ & $94.4444 \%$ \\
5 & Overall_Specificity & $98.9899 \%$ & $99.4949 \%$ \\
6 & Overall_Precision & $93.750 \%$ & $96.6667 \%$ \\
7 & Overall_F1 Score & $88.5317 \%$ & $93.7500 \%$ \\
\hline
\end{tabular}

\subsubsection{Experiment 5}

The goal of this experiment is to compare the results of our method with that of reference [9], which is one of the state-of-the-art methods for ECG identification. Table 9 shows the comparison between reference [9] and the proposed method.

Table 9. Comparison of the proposed method with reference [9] (SI-Subject Identification, HI-Heartbeat Identification).

\begin{tabular}{ccccc}
\hline \multirow{2}{*}{ Methods } & \multicolumn{2}{c}{ ECG-ID: 89 } & \multicolumn{2}{c}{ MIT-BIH: 47 } \\
\cline { 2 - 5 } & $\begin{array}{c}\text { ECG Length } \\
\text { (in Number of Heartbeats) }\end{array}$ & SI & $\begin{array}{c}\text { ECG Length } \\
\text { (in Number of Heartbeats) }\end{array}$ & SI \\
\hline RNN [9] & 18 & $91.7 \%$ & 18 & $93.3 \%$ \\
GRU [9] & 18 & $94.4 \%$ & 18 & $95.7 \%$ \\
LSTM [9] & 18 & $100 \%$ & 18 & $100 \%$ \\
Proposed method & 18 & $97.75 \%$ & 18 & $100 \%$ \\
Proposed method & 7 & $95.25 \%$ & 7 & $97.85 \%$ \\
Proposed method & 5 & $92.36 \%$ & 5 & $97.80 \%$ \\
Proposed method & 3 & $89.97 \%$ & 3 & $96.96 \%$ \\
Proposed method & 1 & $84.50 \%$ & 1 & $90.45 \%$ \\
\hline
\end{tabular}

Results on Table 9 show that LSTM network performs better than GRU and traditional RNN in terms of the subject identification accuracy, and that our approach behaves similar to the LSTM network. On the databases, ECG-ID, and the MITDB, subject identification accuracies of $97.75 \%$ and $100 \%$ were achieved. Besides that, the proposed method could achieve high heartbeat identification accuracy of $84.5 \%$ and $90.45 \%$ on ECG-ID database and the MITDB, which made it possible to obtain good performance based on short-term signals. For ECG-ID and the MITDB, $95.25 \%$ and $97.85 \%$ subject identification accuracy were obtained even with signals of only seven-heartbeat length. Especially, the subject identification accuracy of signals of three-heartbeat length could reach $96.96 \%$ on the MITDB.

These results further proved the effectiveness of our method for short-term ECG signal identification.

Table 10 shows the state-of-the-art methods proposed for ECG biometric identification. Compared with other methods, the proposed method achieves high-level results in terms of subject identification accuracy on both databases, which suggests that it can serve as an effectively partial-fiducial way for ECG biometric identification. 
Table 10. Performance comparison with state-of-the-art works (SI-Subject Identification).

\begin{tabular}{|c|c|c|c|c|c|}
\hline Methods & Year & Feature Extraction (Type) & Decision & ECG Dataset & Performance \\
\hline Page et al. [12] & 2015 & QRS complex segments (Fiducial) & NN & ECG-ID: 90 & SI: $99.93 \%$ \\
\hline Dar et al. [40] & 2015 & $\begin{array}{l}\text { Haar Transform/GBFS } \\
\text { (Non-fiducial) }\end{array}$ & KNN & $\begin{array}{l}\text { ECG-ID: } 90 \\
\text { MIT-BIH: } 47\end{array}$ & $\begin{array}{l}\text { SI: } 83.2 \% \\
\text { SI: } 95.9 \%\end{array}$ \\
\hline Dar et al. [10] & 2015 & $\begin{array}{c}\text { Haar Transform and HRV/GBFS } \\
\text { (Non-fiducial) }\end{array}$ & Random Forest & $\begin{array}{l}\text { ECG-ID: } 90 \\
\text { MIT-BIH: } 47\end{array}$ & $\begin{array}{l}\text { SI: } 83.9 \% \\
\text { SI: } 93.1 \%\end{array}$ \\
\hline Dhou-ha et al. [41] & 2016 & $\begin{array}{l}21 \text { temporal and amplitude features } \\
\text { and } 10 \text { morphological descriptors } \\
\text { (Fiducial) }\end{array}$ & SVM & MIT-BIH: 44 & SI: $98.8 \%$ \\
\hline Tan et al. [42] & 2017 & $\begin{array}{l}\text { Temporal, amplitude, and angle fid. } \\
\text { + DWT coefficients (Fiducial) }\end{array}$ & $\begin{array}{l}\text { Random } \\
\text { Forests + } \\
\text { WDIST KNN }\end{array}$ & $\begin{array}{l}\text { ECG-ID: } 89 \\
\text { MIT-BIH: } 47\end{array}$ & $\begin{array}{l}\text { SI: } 100 \% \\
\text { SI: } 100 \%\end{array}$ \\
\hline Yu et al. [43] & 2017 & PCA (Non-fiducial) & RPROP & ECG-ID: 89 & SI: $96.60 \%$ \\
\hline Lynn et al. [44] & 2018 & $\begin{array}{l}\text { Temporal and amplitude features } \\
\text { (Fiducial) }\end{array}$ & BP-NN & $\begin{array}{l}\text { ECG-ID: } 3 \\
10 \\
20\end{array}$ & $\begin{array}{l}\text { SI: } 98.24 \% \\
\text { SI: } 96.20 \% \\
\text { SI: } 94.00 \%\end{array}$ \\
\hline Zhao et al. [45] & 2018 & $\begin{array}{c}\text { Generalized S-transformation } \\
\text { (Non-fiducial) }\end{array}$ & CNN & ECG-ID:50 & SI: $96.63 \%$ \\
\hline Mahmoud et al. [46] & 2018 & $\begin{array}{l}\text { Mean P-QRS-T fragment + DWT } \\
\text { (Fusion of fiducial and non-fiducial) }\end{array}$ & SVM & $\begin{array}{l}\text { ECG-ID: } 90 \\
\text { MIT-BIH: } 47\end{array}$ & $\begin{array}{l}\text { SI: } 99 \% \\
\text { SI: } 100 \%\end{array}$ \\
\hline Proposed Method & - & $\begin{array}{l}\text { QRS-centered resampling strategy + } \\
\text { PCANet (partial-fiducial) }\end{array}$ & Linear-SVM & $\begin{array}{l}\text { ECG-ID: } 12 \\
89 \\
\text { MIT-BIH: } 47\end{array}$ & $\begin{array}{l}\text { SI: } 97.78 \% \\
\text { SI: } 97.75 \% \\
\text { SI: } 100 \%\end{array}$ \\
\hline
\end{tabular}

\section{Discussion}

In this work, a novel HR robust method for short-term ECG biometric identification was developed. Raw ECG signals were filtered with wavelet denoising and segmented into heartbeats by taking the detected $\mathrm{T}$ peak points as delimiters. Then the heartbeat was processed by the proposed QRS-centered resampling strategy and standardized to 400 sampling points. The QRS-centered strategy is inspired and based on the prior ECG identification works: Firstly, to our knowledge, all the existing literature about ECG identification has taken QRS complex or its related form as features and QRS complex is very important for identifying a person $[9,10,12,18,40-46]$. To preserve all potential identity information of the QRS complex, we use a length-fixed window to keep the QRS complex original. Secondly, it is also found that mapping the heartbeat into a regular interval of segments does help to deal with the HR variability problem $[5,20]$. Thus, we segment the heartbeat into three parts, which are the first, the QRS and the third part respectively, and unify them. In this way, the extracted heartbeat can correct $\mathrm{T}$ wave shift without using traditional QT correction formula, and contain sufficient information of QRS such as QRS negative area, amplitude, and so on at the same time. As a result, HR variability is removed and more potential information is provided for the subsequent steps, which is beneficial for the identification accuracy.

After that, PCANet was implemented to learn discrimination among heartbeats from different persons by taking the principal eigenvectors as filter banks. PCANet can be analyzed by comparing it with the Convolutional Neural Network (CNN): Like CNN, the PCANet also has convolution filter bank in each stage; the binary quantization of the PCANet at the output stage performs similar function like the nonlinear layer in CNN; the pooling layer of the PCANet is set to be the block-wise histograms of binary codes. In fact, PCANet can be essentially considered as a CNN model, which has strong capability of feature extraction $[26,47,48]$.

Moreover, the PCANet seems to be more likely to achieve better performance than traditional CNN in ECG biometric identification, especially for ECG data without outlier correction. As we all know, ECG is not a strict periodic signal, and local distortions and variation on heartbeats may appear even without HR variability. Traditional CNN may be influenced by these distortions because its convolutional filter bank is learned in a data-adapting way. Compared with traditional CNN, the filter bank of the PCANet is prefixed by analyzing the main difference between subjects based on the combination of patch matrices of the training data. This learning way gives more holistic observations of the original ECG data, and the learned intra-invariance can essentially capture more identification 
information $[26,47,48]$. According to our experimental results, the PCANets did obtain much higher heartbeat identification than the CNNs with different heartbeat resampling manner, which further proved that the PCANet method was robust to distortions.

Compared with other methods in literature, our method has two main advantages. First, the method is HR robust and does not require HR based QT correction. HR variability can be removed under short-term ECG signal condition and avoid the complex operations of accurate Q detection or HR measurement. Based on the experiments, it is found that not only the amplitude, but also the temporal and morphological information of QRS can potentially contribute to identification, which is in accordance with other studies $[5,20,21]$. Second, the proposed method can achieve high heartbeat identification accuracy, which makes it suitable for systems that use a small quantity of heartbeats to make a decision [49]. Besides that, the naturality of PCANet makes our work easy to be reproduced by other researchers. Because compared with traditional neural networks $(\mathrm{CNN})$, only a few parameters are required to determine a PCANet $[25,26,48]$.

In the future, we will explore the "other class" classification problem. "Other class" refers to the class that classifier has not yet trained and it is totally different from the other trained individuals morphologically. In ECG identification, we can also call it unknown individual. The classification of the unknown individual is an open-set problem and can hardly be solved by some simple methods such as threshold setting [50] or distance matching [51]. So our next stage research is to improve our existing identification architecture and realize "other class" recognition.

\section{Conclusions}

In this paper, we propose a novel HR robust method for short-term ECG signal identification. In this study, we identified ECG signals by using the QRS-centered resampling strategy and the PCANet. To evaluate the effectiveness of our algorithm, the experiment was performed on ECG fragments with different length and various HRs. Experimental results revealed that this QRS-centered resampling strategy could efficiently remove the influence of HR variability and the PCANet was able to capture important information required for class discrimination from processed heartbeats. Compared with the existing state-of-the-art methods, the proposed approach provides an effectively partial-fiducial way for identification and shows comparative results on both ECG-ID database and the MITDB. Our method is expected to contribute to information security and privacy protection.

Author Contributions: Conceptualization-D.W. and Y.S., Data curation-W.Y. and G.Z., Formal analysis-D.W. and T.L., Writing-Original Draft D.W. and Y.S., Writing-Edit and Review, D.W. and W.Y.

Funding: This work was supported by the Key Scientific and Technological Research Project of Jilin Province under Grant No. 20170414017GH and 20190302035GX; the Natural Science Foundation of Guangdong Province under Grant No. 2016A030313658; the Premier-Discipline Enhancement Scheme Supported by Zhuhai Government under Grant No. 2015YXXK02-2; the Premier Key-Discipline Enhancement Scheme Supported by Guangdong Government Funds under Grant No. 2016GDYSZDXK036; Science Foundation of China under Grant No. 61741311; Natural Science Foundation of China under Grant No. 61702249.

Conflicts of Interest: The authors declare no conflict of interest.

\section{References}

1. Komeili, M.; Armanfard, N.; Hatzinakos, D. Liveness Detection and Automatic Template Updating Using Fusion of ECG and Fingerprint. IEEE Trans. Inf. Forensics Secur. 2018, 13, 1810-1822. [CrossRef]

2. Belgacem, N.; Fournier, R.; Nait-Ali, A.; Bereksi-Reguig, F. A novel biometric authentication approach using ECG and EMG signals. J. Med. Eng. Technol. 2015, 39, 226-238. [CrossRef] [PubMed]

3. Fang, S.C.; Chan, H.L. QRS detection-free electrocardiogram biometrics in the reconstructed phase space. Pattern Recognit. Lett. 2013, 34, 595-602. [CrossRef]

4. Karimian, N.; Guo, Z.M.; Tehranipoor, M.; Forte, D. Highly Reliable Key Generation From Electrocardiogram (ECG). IEEE Trans. Biomed. Eng. 2017, 64, 1400-1411. [CrossRef] 
5. Tuerxunwaili; Nor, R.M.; Rahman, A.W.B.A.; Sidek, K.A.; Ibrahim, A.A. Electrocardiogram Identification: Use a Simple Set of Features in QRS Complex to Identify Individuals. In Proceedings of the 12th International Conference on Computing and Information Technology (IC2IT), Khon-Kaen, Thailand, 7-8 July 2016; Springer: Cham, Switzerland, 2016; pp. 139-148. [CrossRef]

6. Ghongade, R.; Ghatol, A. An effective feature set for ECG pattern classification. In Proceedings of the International Conference on Medical Biometrics, Hong Kong, China, 4-5 January 2008; pp. 25-32. [CrossRef]

7. Liu, S.-H.; Cheng, D.-C.; Lin, C.-M. Arrhythmia Identification with Two-Lead Electrocardiograms Using Artificial Neural Networks and Support Vector Machines for a Portable ECG Monitor System. Sensors 2013, 13, 813-828. [CrossRef] [PubMed]

8. Zhao, Z.; Yang, L.; Chen, D.; Luo, Y. A Human ECG Identification System Based on Ensemble Empirical Mode Decomposition. Sensors 2013, 13, 6832-6864. [CrossRef] [PubMed]

9. Salloum, R.; Kuo, C.C.J. ECG-based biometrics using recurrent neural networks. In Proceedings of the IEEE International Conference on Acoustics, Speech and Signal Processing (ICASSP), New Orleans, LA, USA, 5-9 March 2017; pp. 2062-2066. [CrossRef]

10. Dar, M.N.; Akram, M.U.; Usman, A.; Khan, S.A. ECG Biometric Identification for General Population Using Multiresolution Analysis of DWT Based Features. In Proceedings of the Second International Conference on Information Security and Cyber Forensics (InfoSec), Cape Town, South Africa, 15-17 November 2015; pp. 5-10. [CrossRef]

11. Tseng, K.-K.; Lee, D.; Hurst, W.; Lin, F.-Y.; Ip, W.H. Frequency Rank Order Statistic with Unknown Neural Network for ECG Identification System. In Proceedings of the 4th International Conference on Enterprise Systems (ES), Melbourne, VIC, Australia, 2-3 November 2016; pp. 160-167. [CrossRef]

12. Page, A.; Kulkarni, A.; Mohsenin, T. Utilizing Deep Neural Nets for an Embedded ECG-based Biometric Authentication System. In Proceedings of the 2015 IEEE Biomedical Circuits and Systems Conference (BioCAS), Atlanta, GA, USA, 22-24 October 2015; pp. 346-349. [CrossRef]

13. Peimankar, A.; Puthusserypady, S. Ensemble Learning for Detection of Short Episodes of Atrial Fibrillation. In Proceedings of the 2018 26th European Signal Processing Conference (EUSIPCO), Roma, Italy, 3-7 September 2018; pp. 66-70. [CrossRef]

14. Poree, F.; Kervio, G.; Carrault, G. ECG biometric analysis in different physiological recording conditions. Signal Image Video Process. 2016, 10, 267-276. [CrossRef]

15. Nemirko, A.; Lugovaya, T. Biometric human identification based on electrocardiogram. In Proceedings of the XIIIth Russian Conference on Mathematical Methods of Pattern Recognition, Moscow, Russian, 20-26 June 2005; pp. 387-390. [CrossRef]

16. Gargiulo, F.; Fratini, A.; Sansone, M.; Sansone, C. Subject identification via ECG fiducial-based systems: Influence of the type of QT interval correction. Comput. Meth. Prog. Biomed. 2015, 121, 127-136. [CrossRef]

17. Tawfik, M.M.; Selim, H.; Kamal, T. Human identification using time normalized QT signal and the QRS complex of the ECG. In Proceedings of the 7th International Symposium on Communication Systems, Networks \& Digital Signal Processing (CSNDSP 2010), Newcastle upon Tyne, UK, 21-23 July 2010; pp. 755-759.

18. Patro, K.K.; Kumar, P.R. Effective Feature Extraction of ECG for Biometric Application. In Proceedings of the 7th International Conference on Advances in Computing \& Communications (ICACC-2017), Cochin, India, 22-24 August 2017; pp. 296-306. [CrossRef]

19. Chen, C.-L.; Chuang, C.-T. A QRS Detection and R Point Recognition Method for Wearable Single-Lead ECG Devices. Sensors 2017, 17, 1969. [CrossRef]

20. Lee, W.; Kim, S.; Kim, D. Individual Biometric Identification Using Multi-Cycle Electrocardiographic Waveform Patterns. Sensors 2018, 18, 1005. [CrossRef]

21. Matveev, M.; Christov, I.; Krasteva, V.; Bortolan, G.; Simov, D.; Mudrov, N.; Jekova, I. Assessment of the stability of morphological ECG features and their potential for person verification/identification. In Proceedings of the 21st International Conference on Circuits, Systems, Communications and Computers (CSCC 2017), Crete Island, Greece, 14-17 July 2017. [CrossRef]

22. Acharya, U.R.; Fujita, H.; Lih, O.S.; Hagiwara, Y.; Tan, J.H.; Adam, M. Automated detection of arrhythmias using different intervals of tachycardia ECG segments with convolutional neural network. Inf. Sci. 2017, 405, 81-90. [CrossRef]

23. Acharya, U.R.; Oh, S.L.; Hagiwara, Y.; Tan, J.H.; Adam, M.; Gertych, A.; Tan, R.S. A deep convolutional neural network model to classify heartbeats. Comput. Biol. Med. 2017, 89, 389-396. [CrossRef] [PubMed] 
24. Andersen, R.S.; Peimankar, A.; Puthusserypady, S. A deep learning approach for real-time detection of atrial fibrillation. Expert Syst. Appl. 2019, 115, 465-473. [CrossRef]

25. Yang, W.; Si, Y.; Wang, D.; Guo, B. Automatic recognition of arrhythmia based on principal component analysis network and linear support vector machine. Comput. Biol. Med. 2018, 101, 22-32. [CrossRef] [PubMed]

26. Chan, T.-H.; Jia, K.; Gao, S.; Lu, J.; Zeng, Z.; Ma, Y. PCANet: A Simple Deep Learning Baseline for Image Classification? IEEE Trans. Image Process. 2015, 24, 5017-5032. [CrossRef] [PubMed]

27. Jané, R.; Laguna, P.; Thakor, N.V.; Caminal, P. Adaptive baseline wander removal in the ECG: Comparative analysis with cubic spline technique. In Proceedings of the Computers in Cardiology, Durham, NC, USA, 11-14 October 1992; pp. 143-146. [CrossRef]

28. Date, A.A.; Ghongade, R.B. Performance of Wavelet Energy Gradient Method for QRS Detection. In Proceedings of the 4th International Conference on Intelligent and Advanced Systems (ICIAS2012), Kuala Lumpur, Malaysia, 12-14 June 2012; pp. 876-881. [CrossRef]

29. Rakshit, M.; Das, S. An efficient ECG denoising methodology using empirical mode decomposition and adaptive switching mean filter. Biomed. Signal Process. Control 2018, 40, 140-148. [CrossRef]

30. Li, J.; Si, Y.; Lang, L.; Liu, L.; Xu, T. A Spatial Pyramid Pooling-Based Deep Convolutional Neural Network for the Classification of Electrocardiogram Beats. Appl. Sci.-Basel 2018, 8, 1590. [CrossRef]

31. Donoho, D.L.; Johnstone, J.M. Ideal spatial adaptation by wavelet shrinkage. Biometrika 1994, 81, 425-455. [CrossRef]

32. Yao, C.; Si, Y. ECG P, T wave complex detection algorithm based on lifting wavelet. J. Jilin. U Techno Ed. 2013, 43, 177-182. [CrossRef]

33. Goldberger, A.L.; Amaral, L.A.; Glass, L.; Hausdorff, J.M.; Ivanov, P.C.; Mark, R.G.; Mietus, J.E.; Moody, G.B.; Peng, C.-K.; Stanley, H.E. PhysioBank, PhysioToolkit, and PhysioNet: Components of a new research resource for complex physiologic signals. Circulation 2000, 101, e215-e220. [CrossRef]

34. Wei, J.J.; Chang, C.J.; Chou, N.K.; Jan, G.J. ECG data compression using truncated singular value decomposition. IEEE Trans. Biomed. Eng. 2001, 5, 290-299. [CrossRef]

35. PCANet Code. Available online: http://mx.nthu.edu.tw/ \{\}tsunghan (accessed on 5 December 2018).

36. Rumelhart, D.E.; Hinton, G.E.; Williams, R.J. Learning representations by back-propagating errors. Nature 1986, 323, 533-536. [CrossRef]

37. Breiman, L. Random forests. Mach. Learn. 2001, 45, 5-32. [CrossRef]

38. Naive Bayes Code. Available online: https://github.com/andreeas26/NaiveBayesClassifier-Matlab (accessed on 28 December 2018).

39. Fan, R.-E.; Chang, K.-W.; Hsieh, C.-J.; Wang, X.-R.; Lin, C.-J. LIBLINEAR: A Library for Large Linear Classification. J. Mach. Learn. Res. 2008, 9, 1871-1874. [CrossRef]

40. Dar, M.N.; Akram, M.U.; Shaukat, A.; Khan, M.A. Ieee. ECG Based Biometric Identification for Population with Normal and Cardiac Anomalies Using Hybrid HRV and DWT Features. In Proceedings of the 2015 5th International Conference on IT Convergence and Security (ICITCS), Kuala Lumpur, Malaysia, 24-27 August 2015. [CrossRef]

41. Rezgui, D.; Lachiri, Z. ECG Biometric Recognition Using SVM-Based Approach. IEEJ Trans. Electr. Electron. Eng. 2016, 11, S94-S100. [CrossRef]

42. Tan, R.; Perkowski, M. ECG Biometric Identification Using Wavelet Analysis Coupled with Probabilistic Random Forest. In Proceedings of the 2016 15th IEEE International Conference on Machine Learning and Applications (ICMLA), Anaheim, CA, USA, 18-20 December 2016; pp. 182-187. [CrossRef]

43. Yu, J.; Si, Y.; Liu, X.; Wen, D.; Luo, T.; Lang, L. ECG Identification Based on PCA-RPROP. In Proceedings of the International Conference on Digital Human Modeling and Applications in Health, Safety, Ergonomics and Risk Management, Vancouver, BC, Canada, 9-14 July 2017; pp. 419-432. [CrossRef]

44. Lynn, H.M.; Yeom, S.; Kim, P. ECG-based biometric human identification based on backpropagation neural network. In Proceedings of the 2018 Conference Research in Adaptive and Convergent Systems (RACS 2018), Honolulu, HI, USA, 9-12 October 2018; pp. 6-10. [CrossRef]

45. Zhao, Z.; Zhang, Y.; Deng, Y.; Zhang, X. ECG authentication system design incorporating a convolutional neural network and generalized S-Transformation. Comput. Biol. Med. 2018, 102, 168-179. [CrossRef]

46. Bassiouni, M.M.; El-Dahshan, E.-S.A.; Khalefa, W.; Salem, A.M. Intelligent hybrid approaches for human ECG signals identification. Signal Image Video Process. 2018, 12, 941-949. [CrossRef] 
47. Sun, Z.; Chiong, R.; Hu, Z.-P. An extended dictionary representation approach with deep subspace learning for facial expression recognition. Neurocomputing 2018, 316, 1-9. [CrossRef]

48. Wu, J.; Qiu, S.; Kong, Y.; Jiang, L.; Chen, Y.; Yang, W.; Senhadji, L.; Shu, H. PCANet: An energy perspective. Neurocomputing 2018, 313, 271-287. [CrossRef]

49. Chun, S.Y. Single Pulse ECG-based Small Scale User Authentication using Guided Filtering. In Proceedings of the IEEE International Conference on Biometrics (ICB), Halmstad, Sweden, 13-16 June 2016. [CrossRef]

50. Bendale, A.; Boult, T.E. Towards Open Set Deep Networks. In Proceedings of the IEEE Conference on Computer Vision and Pattern Recognition (CVPR), Las Vegas, NV, USA, 27-30 June 2016; pp. 1563-1572. [CrossRef]

51. Mendes Junior, P.R.; de Souza, R.M.; Werneck, R.d.O. Nearest neighbors distance ratio open-set classifier. Mach. Learn. 2017, 106, 359-386. [CrossRef]

(C) 2019 by the authors. Licensee MDPI, Basel, Switzerland. This article is an open access article distributed under the terms and conditions of the Creative Commons Attribution (CC BY) license (http://creativecommons.org/licenses/by/4.0/). 